\title{
CONDITIONS FOR SELF-SYNCHRONIZATION OF INERTIAL VIBRATORS OF VIBRATORY CONVEYORS IN GENERAL MOTION ${ }^{1}$
}

\author{
Grzegorz Cieplok, Kaja Wójcik \\ AGH University of Science and Technology, The Faculty of Mechanical Engineering and Robotics, Krakow, Poland \\ e-mail: cieplok@agh.edu.pl; kwojcik@agh.edu.pl
}

\begin{abstract}
The paper concerns the analysis of a self-synchronization process of inertial vibrators in general motion. These types of systems are found e.g. in the case of vibratory conveyors, in which the synchronization plane of vibrators is perpendicular to the plane of vibration of the conveyor trough. The analysis is focused on two basic issues related to movement of the vibrator. The first issue concerns conditions for obtaining stable motion in a configuration ensuring generation of useful vibrations. The second one is determination of the value of the synchronizing moment. In order to obtain analytical dependencies as simply as possible, we considered a typical build conveyor and the suspension, whose mathematical description we can bring to a diagonal form.

In the paper, we paid attention to the consequences of using suspensions with highly directional properties, e.g. metal-elastomer vibration isolators.
\end{abstract}

Keywords: vibratory machines, self-synchronization, general motion, metal-elastomer vibro-isolators

\section{Introduction}

The issues of self-synchronization of inertial vibrators is a well-recognized area of machine dynamics. Extensive studies concerning synchronization of dynamic systems we can find in papers by Blechman (1971, 2000), specific applications in papers, e.g. Michalczyk (2012), Michalczyk and Cieplok (2014) and more (Hou et al., 2017; Michalczyk and Pakuła, 2016; Zhao et al., 2011; Fang et al., 2015; Karmazyn et al., 2018; Francke et al., 2010). The principles of building vibrating machines with a vibratory drive are known (Goncharewich and Frolov, 1990; Michalczyk, 1995; Cieplok, 2009, 2018), in particular conditions of synchronous motion with distinction between working in the desirable and undesirable state. One of the most widespread in the industry type of vibratory machines are machines working over-resonance. We can include vibratory conveyors, bolters, feeders, table vibrators, etc. In the case of machines working over resonance frequencies, the natural vibrations of the machine body-suspension system are definitely lower than angular velocity of vibrators. Basically we can say that already with a difference of four to five times these frequencies, we can ignore the impact of the spring suspension on movement of the machine body. This situation is available by coil springs (Michalczyk and Bera, 2019; Michalczyk, 2017) commonly used in machine suspensions.

The matter may change when the coil spring is replaced by metal-elastomer joints (Sikora et al., 2016, 2018). Depending on the purpose, they may present pronounced directional characteristics. This type of vibro-isolators are intended for working in one plane. Such a situation does not disturb and often helps the machines to direct their movement. An exception may be machines in which the synchronization plane of vibrators is perpendicular to the vibro-isolators working plane. Increased stiffness of the suspension in the synchronization plane leads to a displacement

${ }^{1}$ PCM-CMM 2019, Krakow, Poland, September 8-12, 2019 
of natural frequencies of the system towards the working point. It may have a significant impact on the self-synchronization process of vibrators. The case in point (Fig. 1a) should be considered within the scope of general motion, unlike those (Fig. 1b) in which the self-synchronization plane responds to the working plane. They may be considered as regards to the plane motion.

(a)

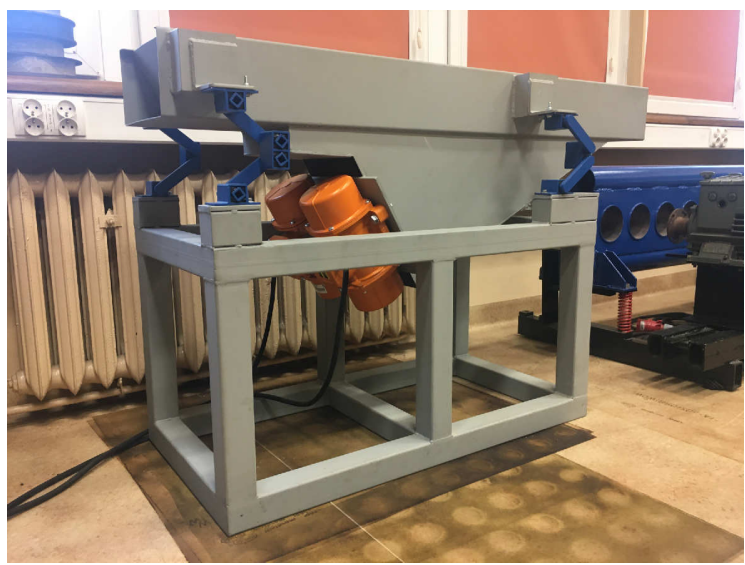

(b)

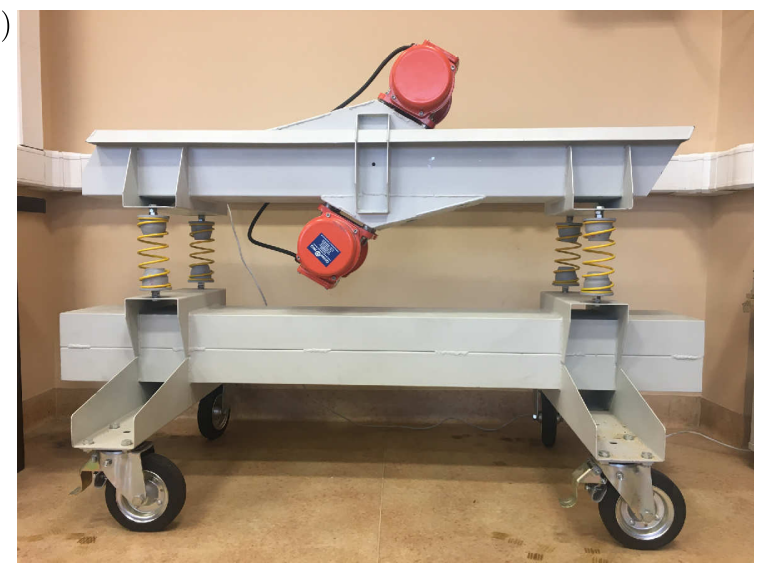

Fig. 1. Examples of vibratory conveyors of different constructions. Vibromechanics Laboratory AGH. (a) Conveyor in general motion, (b) conveyor in plane motion

\section{Model of conveyor}

Let us consider the model of a conveyor presented in Fig. 2. It consists of a body mounted on an elastic suspension induced to vibrate by the use of two inertial vibrators. The vibrator working plane is perpendicular to the working plane of the conveyor trough and passes over the center of gravity of the body. The vibrators are driven counter-rotating by asynchronous motors. The suspension has been simplified to elastic elements operating independently of their coordinates, namely to the system whose mathematical description can be reduced to a diagonal form. The bodies of the system move in general motion, while the movement of the trough body can be considered in the range of small angular displacements.

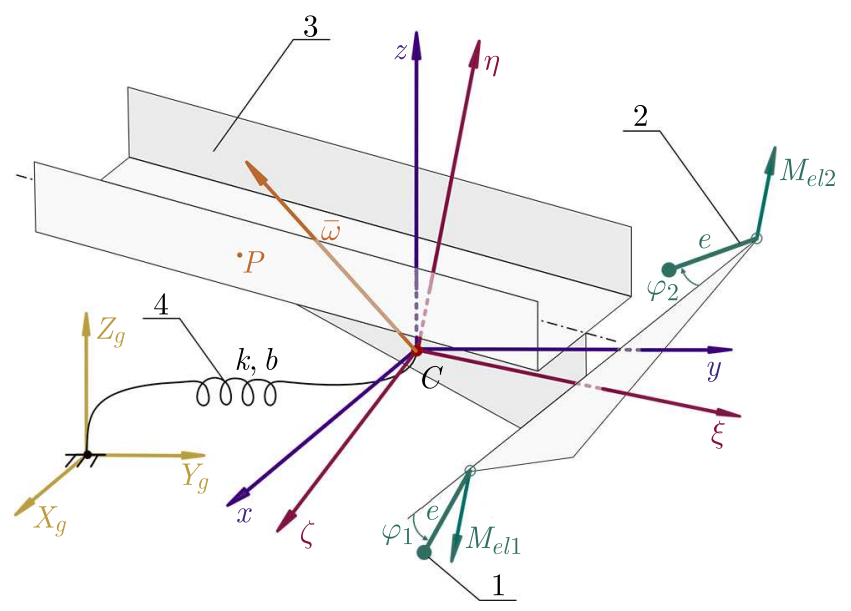

Fig. 2. Phenomenological model of the conveyor examined in the paper; 1,2 - inertial vibrators,

3 - machine body, 4 - elastic suspension, $\bar{\omega}$ - vector of angular solid velocity, $C$ - body center of mass,

$P$ - selected point on the body of the conveyor, $0 X_{g} Y_{g} Z_{g}$ - global coordinate system (fixed),

$C x y z$ - moving system parallel to $0 X_{g} Y_{g} Z_{g}, C \xi \eta \zeta$ - system defined by the principal axes of inertia of the trough body, $M_{e l_{1}}, M_{e l_{2}}$ - drive rotors moments 
The Lagrange equation of the second kind was used to determine dynamic equations of motion. As generalized coordinates, the coordinates of the body mass center $x_{C}, y_{C}, z_{C}$, Euler's angles of the body (Fig. 3a) and two coordinates $\varphi_{1}, \varphi_{2}$ describing the angular positions of the vibrators were assumed. In the case of vibrating motion of rigid bodies, it is more convenient to use a pre-turned Euler angle system. At the paper, two preliminary revolutions were decided: $\theta=\pi / 2$ and $\Psi=\pi / 2$. Then, in the position of equilibrium, the axes of the Cartesian system $C x y z$ match the axes of the $C \zeta \xi \eta$ system associated with the principal axes of inertia of the trough body.

(a)

$\zeta$

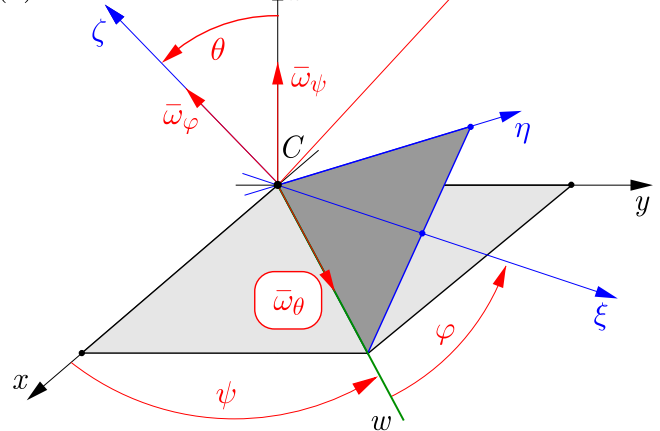

(b)

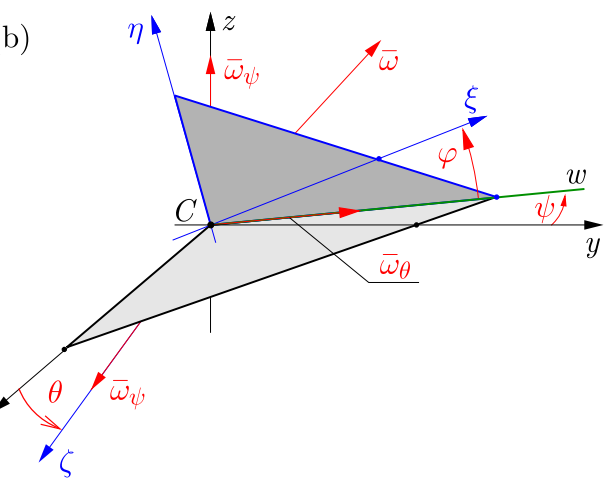

Fig. 3. Coordinates of rotation of a rigid body about a fixed point: (a) Euler's angles in the traditional system, (b) Euler's angle system after revolutions of $\theta=\pi / 2$ and $\Psi=\pi / 2$

We can simply put the coordinates of the vibrators centers of masses in the system $C \xi \eta \zeta$ as

$$
\xi_{1,2}=\xi_{10} \pm e \sin \left(\varphi_{1,2}\right) \quad \zeta_{1,2}= \pm \zeta_{10}+e \cos \left(\varphi_{1,2}\right) \quad \eta_{1,2}=\eta_{10}
$$

where: $\xi_{10}, \eta_{10}, \zeta_{10}$ - coordinates of the vibrators axes mounting points.

The transformation between the coordinates of the $C \xi \eta \zeta$ and $C x y z$ systems allowed for the conversion

$$
\left[\begin{array}{l}
x_{P} \\
y_{P} \\
z_{P}
\end{array}\right]=\left[\begin{array}{ccc}
\cos \Psi \sin \varphi \sin \theta-\sin \Psi \cos \varphi & \cos \Psi \cos \varphi \sin \theta+\sin \Psi \sin \varphi & \cos \Psi \cos \theta \\
\sin \Psi \sin \varphi \sin \theta+\cos \Psi \cos \varphi & \sin \Psi \cos \varphi \sin \theta-\cos \Psi \sin \varphi & \sin \Psi \cos \theta \\
\sin \varphi \cos \theta & \cos \varphi \cos \theta & -\sin \theta
\end{array}\right]\left[\begin{array}{c}
\xi_{P} \\
\eta_{P} \\
\zeta_{P}
\end{array}\right]
$$

based on which, considering small angular shifts of the body, we can write

$$
\begin{aligned}
& x_{1,2}= \pm \zeta_{10}-\Psi\left(\xi_{10} \pm e \sin \left(\varphi_{1,2}\right)\right)+\eta_{10} \theta+e \cos \left(\varphi_{1,2}\right) \\
& y_{1,2}=\Psi\left( \pm \zeta_{10}+e \cos \left(\varphi_{1,2}\right)\right)+\xi_{10} \pm e \sin \left(\varphi_{1,2}\right)-\eta_{10} \varphi \\
& z_{1,2}=-\theta\left( \pm \zeta_{10}+e \cos \left(\varphi_{1,2}\right)\right)+\varphi\left(\xi_{10} \pm e \sin \left(\varphi_{1,2}\right)\right)+\eta_{10}
\end{aligned}
$$

We obtained absolute velocities (in $0 X_{g} Y_{g} Z_{g}$ system) of centers of the vibrators masses by time differentiation of (2.3) and considering the system velocity of $0 x y z$. After removal of the small of second order, we obtain

$$
\begin{aligned}
& \dot{x}_{1,2} \cong-\xi_{10} \dot{\Psi}+\eta_{10} \dot{\theta}-e \dot{\varphi}_{1} \sin \left(\varphi_{1,2}\right) \mp e \dot{\Psi} \sin \left(\varphi_{1,2}\right)+\dot{x}_{C} \\
& \dot{y}_{1,2} \cong \pm \zeta_{10} \dot{\Psi} \pm e \dot{\varphi}_{1,2} \cos \left(\varphi_{1,2}\right)+e \dot{\Psi} \cos \left(\varphi_{1,2}\right)-\eta_{10} \dot{\varphi}+\dot{y}_{C} \\
& \dot{z}_{1,2} \cong \mp \zeta_{10} \dot{\theta}+\xi_{10} \dot{\varphi}-e \dot{\theta} \cos \left(\varphi_{1,2}\right) \pm e \dot{\varphi} \sin \left(\varphi_{1,2}\right)+\dot{z}_{C}
\end{aligned}
$$

By using the velocities of individual solids of the system, we can formulate the Lagrange function for it 


$$
\begin{aligned}
\mathcal{L} \cong & \frac{1}{2} m_{k}\left(\dot{x}_{C}^{2}+\dot{y}_{C}^{2}+\dot{z}_{C}^{2}\right)+\frac{1}{2} J_{\xi} \omega_{\xi}^{2}+\frac{1}{2} J_{\eta} \omega_{\eta}^{2}+\frac{1}{2} J_{\zeta} \omega_{\zeta}^{2}+\frac{1}{2} m_{w}\left(\dot{x}_{1}^{2}+\dot{y}_{1}^{2}+\dot{z}_{1}^{2}\right)+\frac{1}{2} J_{w} \omega_{\eta 1}^{2} \\
& +\frac{1}{2} J_{w_{x y}} \omega_{\xi 1}^{2}+\frac{1}{2} J_{w_{x y}} \omega_{\zeta 1}^{2}+\frac{1}{2} m_{w}\left(\dot{x}_{2}^{2}+\dot{y}_{2}^{2}+\dot{z}_{2}^{2}\right)+\frac{1}{2} J_{w} \omega_{\eta 2}^{2}+\frac{1}{2} J_{w_{x y}} \omega_{\xi 2}^{2} \\
& +\frac{1}{2} J_{w_{x y}} \omega_{\zeta 2}^{2}-\left(\frac{1}{2} k_{x} x_{C}^{2}+\frac{1}{2} k_{y} y_{C}^{2}+\frac{1}{2} k_{z} z_{C}^{2}+\frac{1}{2} k_{\Psi} \Psi^{2}+\frac{1}{2} k_{\theta} \theta^{2}+\frac{1}{2} k_{\varphi} \varphi^{2}\right)
\end{aligned}
$$

Due to connection of the vibrators with the trough (schematically shown in Fig. 2), we get: $\omega_{\eta 1}=\dot{\varphi}_{1}+\omega_{\eta}, \omega_{\eta 2}=-\dot{\varphi}_{2}+\omega_{\eta}, \omega_{\xi 1}=\omega_{\xi 2}=\omega_{\xi}, \omega_{\zeta 1}=\omega_{\zeta 2}=\omega_{\zeta}$.

Kinetic energy of the trough and the vibrators stated by using velocity projections on the axes of the system $C \xi \eta \zeta$ written in formula (2.5) must be expressed by means of the generalized coordinates. This transition is made by the following operation

$$
\left[\begin{array}{l}
\omega_{\xi} \\
\omega_{\eta} \\
\omega_{\zeta}
\end{array}\right]=\left[\begin{array}{ccc}
\sin \varphi \cos \theta & \cos \varphi & 0 \\
\cos \varphi \cos \theta & -\sin \varphi & 0 \\
-\sin \theta & 0 & 1
\end{array}\right]\left[\begin{array}{l}
\omega_{\Psi} \\
\omega_{\theta} \\
\omega_{\varphi}
\end{array}\right]
$$

on the basis of which, for small angular displacements, we obtain

$$
\omega_{\xi} \cong \dot{\theta}+\varphi \dot{\Psi} \quad \omega_{\eta} \cong \dot{\Psi}+\varphi \dot{\theta} \quad \omega_{\zeta} \cong \dot{\varphi}-\theta \dot{\Psi}
$$

Dynamic equations of the conveyor motion can then be determined on the base of the Lagrange-Euler equation

$$
\frac{d}{d t} \frac{\partial \mathcal{L}}{\partial \dot{q}_{i}}-\frac{\partial \mathcal{L}}{\partial q_{i}}+\frac{1}{2} \frac{\partial N}{\partial \dot{q}_{i}}=Q_{i}
$$

in which: $q_{i} \in\left\{x_{C}, y_{C}, z_{C}, \Psi, \theta, \varphi, \varphi_{1}, \varphi_{2}\right\}$. In the equations of motion, all generalized forces $Q_{i}$ are zero except for

$$
\begin{aligned}
& Q_{\varphi 1}=M_{e l_{1}}-M_{\text {load }_{1}} \quad Q_{\varphi 2}=M_{e l_{2}}-M_{\text {load }_{2}} \\
& Q_{\Psi}=M_{e l_{2}}-M_{e l_{1}}+M_{\text {load }_{1}}-M_{\text {load }_{2}}
\end{aligned}
$$

The linear loss power function $N$ engaging the energy dissipation in the suspension components can be represented by

$$
N=b_{x} \dot{x}_{C}^{2}+b_{y} \dot{y}_{C}^{2}+b_{z} \dot{z}_{C}^{2}+b_{\Psi} \dot{\Psi}^{2}+b_{\theta} \dot{\theta}^{2}+b_{\varphi} \dot{\varphi}^{2}
$$

Due to extensive form of the dynamic equations of motion and for the sake of brevity of the publication, their explicit form is not presented. A simplified form resulting in the adoption of constant angular velocities of the vibrators is presented. It allows sufficient study of issues related to the conditions of vibrators synchronization.

The conveyor equations of motion for the constant angular velocities of the vibrators assume the form

$$
\begin{aligned}
& \ddot{x}_{C}\left(m_{k}+2 m_{w}\right)-2 m_{w} \xi_{10} \ddot{\Psi}+2 m_{w} \eta_{10} \ddot{\theta}=m_{w} e \omega_{1}^{2} \cos \varphi_{1}+m_{w} e \omega_{2}^{2} \cos \varphi_{2}-k_{x} x_{C} \\
& \ddot{y}_{C}\left(m_{k}+2 m_{w}\right)-2 m_{w} \eta_{10} \ddot{\varphi}=m_{w} e \omega_{1}^{2} \sin \varphi_{1}-m_{w} e \omega_{2}^{2} \sin \varphi_{2}-k_{y} y_{C} \\
& \ddot{z}_{C}\left(m_{k}+2 m_{w}\right)-2 m_{w} \xi_{10} \ddot{\varphi}=-k_{z} z_{C}
\end{aligned}
$$

and

$$
\begin{aligned}
& -2 m_{w} \xi_{10} \ddot{x}_{C}+\left[2 m_{w}\left(\zeta_{10}^{2}+\xi_{10}^{2}+e^{2}\right)+J_{\eta}\right] \ddot{\Psi}-2 m_{w} \eta_{10} \xi_{10} \ddot{\theta} \\
& \quad=m_{w} e\left(\omega_{2}^{2} \sin \varphi_{2}+\omega_{1}^{2} \sin \varphi_{1}\right) \zeta_{10}-m_{w} e\left(\omega_{2}^{2} \cos \varphi_{2}+\omega_{1}^{2} \cos \varphi_{1}\right) \xi_{10}-k_{\Psi} \Psi \\
& 2 m_{w} \eta_{10} \ddot{x}_{C}-2 m_{w} \xi_{10} \eta_{10} \ddot{\theta}+2 m_{w}\left(\eta_{10}^{2}+\zeta_{10}^{2}+J_{\xi}+2 J_{x y}\right) \ddot{\theta} \\
& \quad=2 m_{w} e \eta_{10}\left(\omega_{1}^{2} \cos \varphi_{1}+\omega_{2}^{2} \cos \varphi_{2}\right)-k_{\theta} \theta \\
& 2 m_{w} \xi_{10} \ddot{z}_{C}+\left(2 m_{w} \xi_{10}^{2}+2 J_{x y}+J_{\zeta}\right) \ddot{\psi}=m_{w} e \eta_{10}\left(\omega_{2}^{2} \sin \varphi_{2}-\omega_{1}^{2} \sin \varphi_{1}\right)-k_{\varphi} \varphi
\end{aligned}
$$


Let us consider a typical conveyor, i.e., of a symmetrical design in which the plane of action of the vibrators forces passes through the center of mass of the conveyor in the state of synchronous running. Then

$$
\omega_{1}=\omega_{2}=\omega-\mathrm{const} \quad \varphi_{1}=\omega t+\varphi_{10} \quad \varphi_{2}=\omega t+\varphi_{20}
$$

and equations (2.11) and (2.12) are reduced to a system of three equations

$$
\begin{aligned}
& \ddot{x}_{C}\left(m_{k}+2 m_{w}\right)-2 m_{w} \xi_{10} \ddot{\Psi}=m_{w} e \omega^{2} \cos \left(\varphi_{1}\right)+m_{w} e \omega^{2} \cos \varphi_{2}-k_{x} x_{C} \\
& \ddot{y}_{C}\left(m_{k}+2 m_{w}\right)=m_{w} e \omega^{2} \sin \left(\varphi_{1}\right)-m_{w} e \omega^{2} \sin \left(\varphi_{2}\right)-k_{y} y_{C} \\
& \quad-2 m_{w} \xi_{10} \ddot{x}_{C}+\left[2 m_{w}\left(\zeta_{10}^{2}+\xi_{10}^{2}+e^{2}\right)+J_{\eta}\right] \ddot{\Psi} \\
& \quad=m_{w} e\left(\omega^{2} \sin \varphi_{2}+\omega^{2} \sin \varphi_{1}\right) \zeta_{10}-m_{w} e\left(\omega^{2} \cos \varphi_{2}+\omega^{2} \cos \varphi_{1}\right) \xi_{10}-k_{\Psi} \Psi
\end{aligned}
$$

Other equations, due to the lack of conjugation of coordinates $x_{C}, y_{C}, \Psi$ and the lack of extortion from the vibrators - are neutralized. The conveyor with symmetrical design and extortion through the center of mass was reduced to a plane system.

System of equations (2.14) is solvable. Due to the linear character and harmonic type of extortion, solutions describing the steady state can be determined with the use of a complex transformation. The results obtained in this way can be presented in the form

$$
\begin{aligned}
& A_{x}=m_{w} e \omega^{2} \frac{m_{w} L^{2} \omega^{2} \sin 2 \beta-\mathrm{i}\left(2 m_{w} L^{2} \omega^{2} \cos ^{2} \beta-J_{\eta}^{*} \omega^{2}+k_{\Psi}\right)}{\left(m_{k}^{*} \omega^{2}-k_{x}\right)\left(k_{\Psi}-J_{\eta}^{*} \omega^{2}\right)+\left(2 m_{w} L \cos \beta\right)^{2} \omega^{4}}\left(\mathrm{e}^{\mathrm{i} \alpha}+1\right) \\
& A_{y}=\frac{m_{w} e \omega^{2}}{\left(m_{k}^{*} \omega^{2}-k_{y}\right)}\left(\mathrm{e}^{\mathrm{i} \alpha}-1\right) \\
& A_{\psi}=m_{w} e \omega^{2} L \frac{\left(m_{k}^{*} \omega^{2}-k_{x}\right) \sin \beta+\mathrm{i}\left[2 m_{w} \omega^{2}+\left(k_{x}-m_{k}^{*} \omega^{2}\right)\right] \cos \beta}{\left(m_{k}^{*} \omega^{2}-k_{x}\right)\left(k_{\Psi}-J_{\eta}^{*} \omega^{2}\right)+\left(2 m_{w} L \cos \beta\right)^{2} \omega^{4}}\left(\mathrm{e}^{\mathrm{i} \alpha}+1\right)
\end{aligned}
$$

where

$$
\begin{array}{lrr}
m_{k}^{*}=m_{k}+2 m_{w} & J_{\eta}^{*}=J_{\eta}+2 m_{w}\left(\zeta_{10}^{2}+\xi_{10}^{2}+e^{2}\right) \\
\alpha=\varphi_{20}-\varphi_{10} & \xi_{10}=L \cos \beta & \zeta_{10}=L \sin \beta
\end{array}
$$

On the basis of the solutions, it can be concluded that when $\alpha=\pi$, i.e. when the vibrators pass each other on the axis $0 y$, the conveyor makes a segmental movement along the axis $0 y$, in the opposite phase to extort. In the case, when $\alpha=0$, i.e. when the vibrators pass each other on the axis $0 x$, the conveyor makes a plane motion, which is a combination of translational motion along the axis $O x$ and rotary motion around the axis $0 z$.

In Fig. 4 a comparison between the steady state based on the full model and the processes determined on the basis of analytical solutions is shown. The comparison is based on the parameters presented in Table 1.

Table 1. Physical parameters of the conveyor used in simulations

\begin{tabular}{|c|c|c|c|c|c|c|c|c|c|}
\hline Figure & $\begin{array}{c}m_{k} \\
{[\mathrm{~kg}]}\end{array}$ & $\begin{array}{c}m_{w} \\
{[\mathrm{~kg}]}\end{array}$ & $\begin{array}{c}J_{\eta} \\
{\left[\mathrm{kg} \mathrm{m}{ }^{2}\right]}\end{array}$ & $\begin{array}{c}e \\
{[\mathrm{~m}]}\end{array}$ & $\begin{array}{c}L \\
{[\mathrm{~m}]}\end{array}$ & $\begin{array}{c}\beta \\
{[\mathrm{rad}]}\end{array}$ & $\begin{array}{c}k_{x} \\
{[\mathrm{~N} / \mathrm{m}]}\end{array}$ & $\begin{array}{c}k_{y} \\
{[\mathrm{~N} / \mathrm{m}]}\end{array}$ & $\begin{array}{c}k_{\Psi} \\
{[\mathrm{Nm} / \mathrm{rad}]}\end{array}$ \\
\hline \hline 4 & 77.34 & 4.0 & 7.04 & 0.021 & 0.16 & 0.682 & $1.65 \mathrm{E}+05$ & $1.35 \mathrm{E}+04$ & $2.25 \mathrm{E}+04$ \\
$5 \mathrm{a}, 7 \mathrm{~b}, 7 \mathrm{~d}$ & 77.34 & 4.0 & 7.04 & 0.021 & 0.16 & 0.682 & $1.68 \mathrm{E}+05$ & $6.2 \mathrm{E}+04$ & $2.93 \mathrm{E}+04$ \\
$5 \mathrm{~b}, 7 \mathrm{c}$ & 77.34 & 4.0 & 7.04 & 0.021 & 0.16 & 0.682 & $8.4 \mathrm{E}+05$ & $6.2 \mathrm{E}+04$ & $1.37 \mathrm{E}+05$ \\
$7 \mathrm{a}, 7 \mathrm{e}$ & 77.34 & 4.0 & 7.04 & 0.021 & 0.16 & 0.682 & $4.2 \mathrm{E}+04$ & $1.85 \mathrm{E}+04$ & $7.34 \mathrm{E}+03$ \\
\hline
\end{tabular}




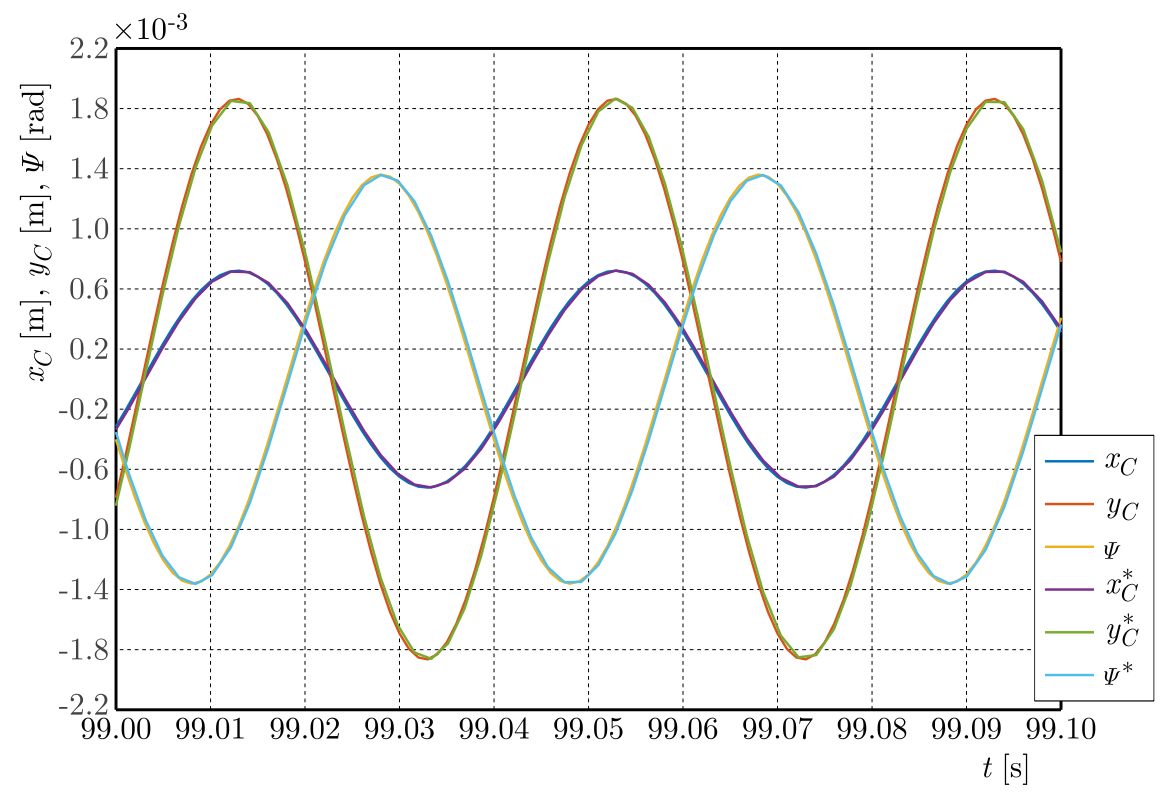

Fig. 4. Steady state - comparison of the full model and analytical solutions. The results of the full model were marked with a star $\left(^{*}\right) ; \Delta M=0.5 \mathrm{Nm}, \alpha=-2.44 \mathrm{rad}$

\section{Stable states of operation}

In steady motion, inertia vibrators can take on two basic states: synchronized and unsynchronized. The first one is a state which guarantees generation of vibrations of one frequency and a constant amplitude. The second one, a disordered state.

The Blekhman criterion is the commonly used approach to determine the conditions of stable motion of vibrators. It allows one to determine the equilibrium position of the oscillating system from the condition of minimum average value of Lagrange's kinetic potential calculated for the period of oscillations of the system

$$
\int_{0}^{T}(E-V) d t=D\left(\varphi_{10}, \varphi_{20}, \ldots, \varphi_{n 0}\right)
$$

The course of the $D$ function depends on the reciprocal position of the vibrators $\varphi_{i 0}$ toward each other. For positions for which the function takes a minimum, the vibrators operate in a stable synchronized state.

For the case considered in the paper, the $D$ function takes the form of

$$
D\left(\varphi_{10}, \varphi_{20}\right)=G \cos \alpha
$$

where

$$
\begin{aligned}
G= & \frac{m_{w}^{2} e^{2} \omega^{4}}{\Delta}\left\{\left[\left(-2 L^{2} \cos (\beta)^{2} m_{w} \omega^{2}+J_{\eta}^{*} \omega^{2}-k_{\Psi}\right)^{2}+L^{4} \sin (2 \beta)^{2} m_{w}^{2} \omega^{4}\right]\left(m_{k}^{*} \omega^{2}-k_{x}\right)\right. \\
& \left.+L^{2}\left[\cos (\beta)^{2}\left(2 m_{w} \omega^{2}-m_{k}^{*} \omega^{2}+k_{x}\right)^{2}+\sin (\beta)^{2}\left(m_{k}^{*} \omega^{2}-k_{x}\right)^{2}\right]\left(J_{\eta}^{*} \omega^{2}-k_{\Psi}\right)\right\} \\
& +\frac{m_{w}^{2} e^{2} \omega^{4}}{k_{y}-m_{k}^{*} \omega^{2}} \\
\Delta= & \left(m_{k}^{*} \omega^{2}-k_{x}\right)\left(k_{\Psi}-J_{\eta}^{*} \omega^{2}\right)+\left(2 m_{w} L \cos \beta\right)^{2} \omega^{4}
\end{aligned}
$$


As we can see, $D$ can only be extreme for two values: $\alpha=0$ or $\alpha=\pi$. The first case corresponds to the situation when counter-rotating vibrators sum up their action in the transverse direction to the movement of the conveyor trough; the second corresponds to the situation when the vibrators sum up their effect in the direction of the conveyor trough.

The stable motion for the desired variant $(\alpha=\pi)$ is therefore obtained when

$$
\operatorname{sgn}(G)=1
$$

Figure 5 presents the course of the function $\operatorname{sgn}(G)$ for two cases - typical, for which the suspension of the machine has a similar stiffness in three directions perpendicular to each other and untypical, with a higher stiffness in a direction transverse to the working plane of the conveyor. It can be seen that already at 5 times higher coefficients $k_{x}$ the range of frequencies of undesirable synchronization is shifted very clearly in the direction of the working point of the machine. We can deal with such a situation in the case of application of steel-elastomer vibro-

(a)

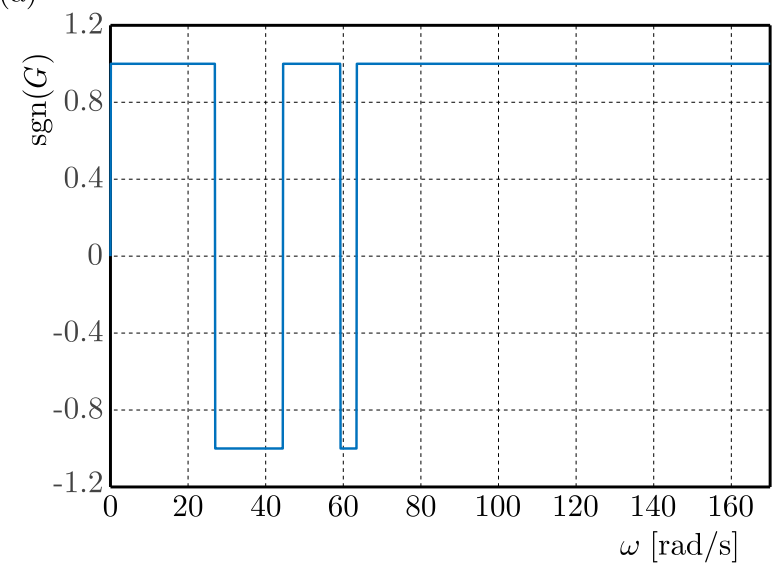

(b)

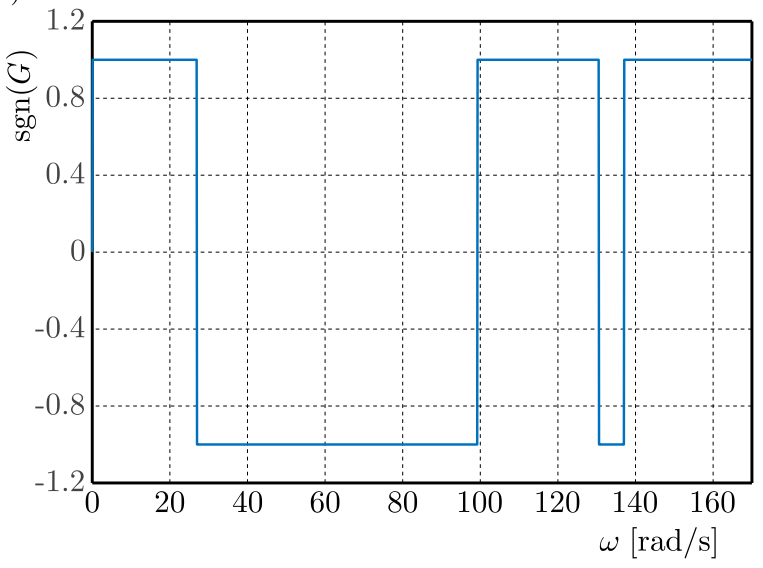

Fig. 5. Influence of suspension stiffness on vibrators synchronization. Value: , $-1 "$ - desirable synchronization, „+1” - undesirable synchronization. (a) Coeficient $k_{x}$ in accordance with Tabele 7 ,

(b) coeficient $k_{x} 5$ times higher with regard to figure (a)

-isolators type ROSTA AB D presented in Fig. 6, which depending on the type, are characterized by strong directional properties. To illustrate the risk, in Fig. 7, the results of conveyor operation

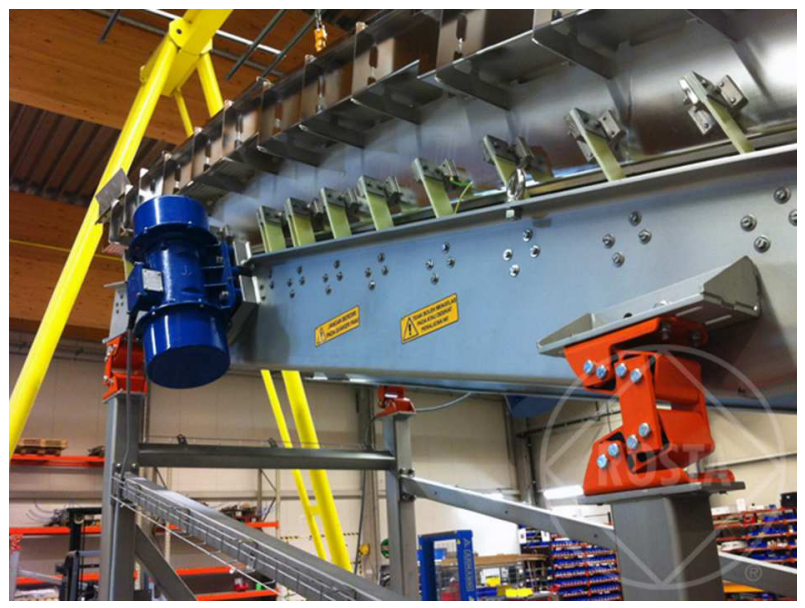

Fig. 6. Example of application of the steel-elastomer joins ROSTA type AB D

at 5 times higher elasticity coefficient $k_{x}$ and the cooperation of the conveyor with the frequency inverter are presented. The use of an inverter is a typical drive solution, which allows one to 
regulate the speed of material movement on the conveyor trough and in specific cases like dosing, including stopping material movement.

(a)

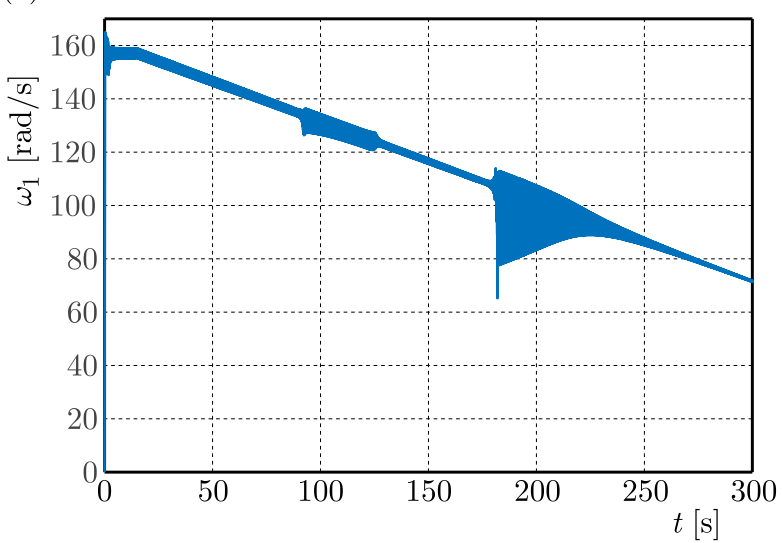

(c)

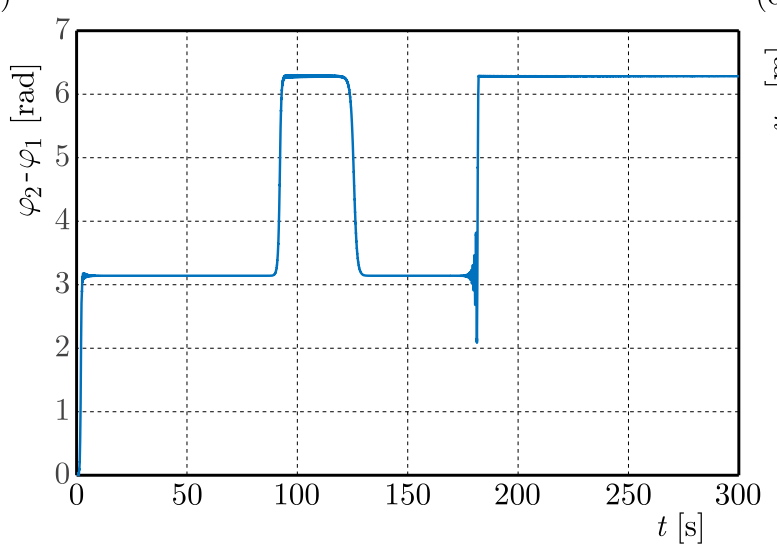

(b)

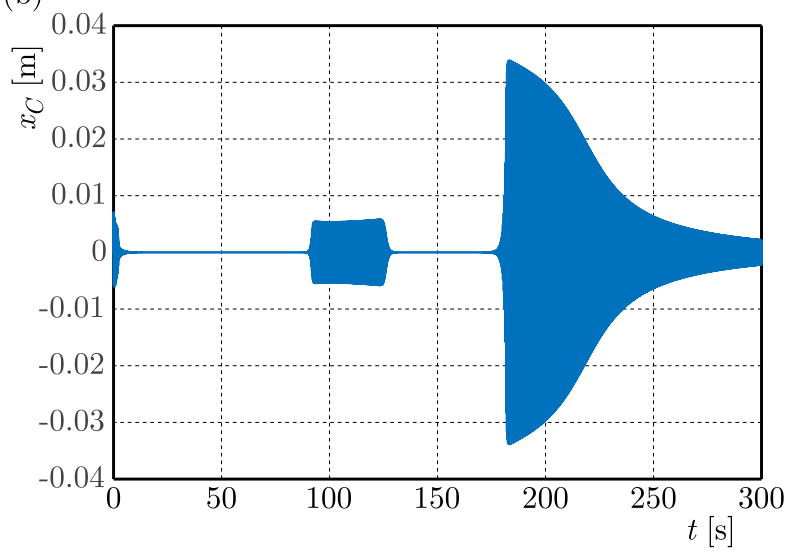

(d)

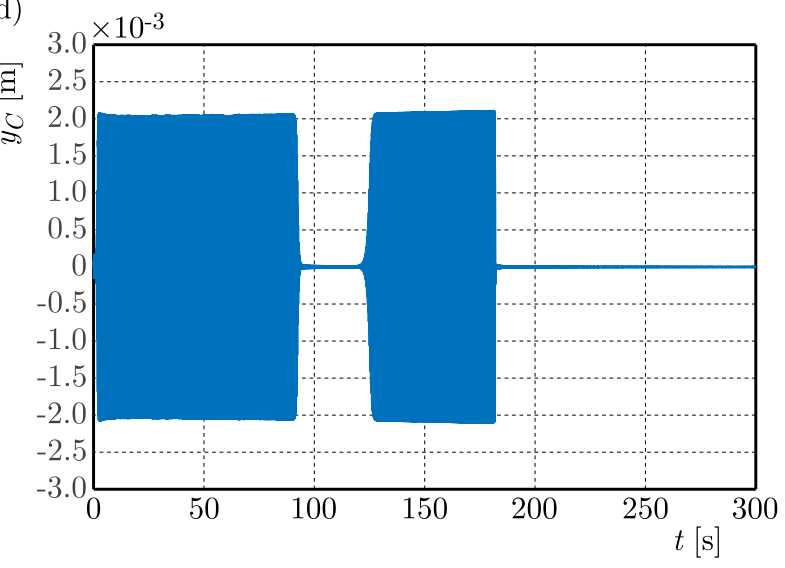

Fig. 7. Coordinates of movement of the conveyor in the case of low damping suspension, $\xi \approx 0.01$.

(a) Angular velocity of the vibrator described by the coordinate $\varphi_{1}$; (b) course of the difference in coordinates of the angular vibrators; the course of $(\mathrm{c}) x_{C}$ coordinate and $(\mathrm{d}) y_{C}$ coordinate of the center of mass of the conveyor body

As we can see, there is a high correlation between the results and the graph shown in Fig. 5 . After the start-up period, the rotors reach a nominal speed of approx. $157.1 \mathrm{rad} / \mathrm{s}$ (Fig. 7a), and in accordance with Fig. 5b they synchronize in the desirable state, Fig. 7b. After the speed has been reduced below $140 \mathrm{rad} / \mathrm{s}$, the configuration of the vibrators is changed to the stable undesirable state. Operation in the undesirable system is carried out at a speed of up to approx. $130 \mathrm{rad} / \mathrm{s}$ and then switch back to the desired configuration. Further decreasing of the rotor angular velocity leads to another change visible at approx. $100 \mathrm{rad} / \mathrm{s}$. The change in the configuration of the vibrators naturally entails a change in the direction of the resultant force of the vibrators and the working direction of the vibrations of the conveyor trough, Fig. 7c,d.

Numerical research based on the full model also allowed one to state the influence of conveyor suspension damping on the process of vibrator synchronization. During lowering the rotation velocity of the vibrators, an increased damping reduced the number of passes between the desired and undesirable states. In particular, in the cases where the operating frequency ranges in a given configuration were narrow. Such a situation is presented in Fig. 8. The relative damping assumed in the simulation at the level of $\xi=0.06$ corresponding to the rubber suspensions. 
(a)

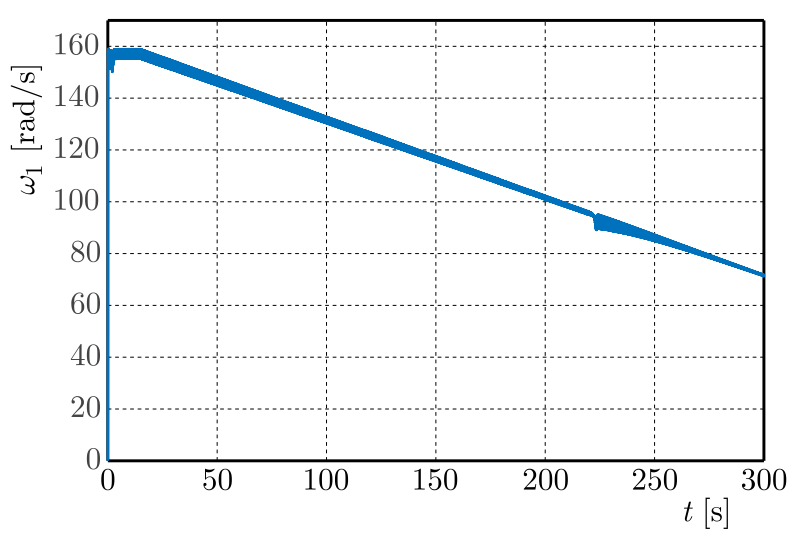

(c)

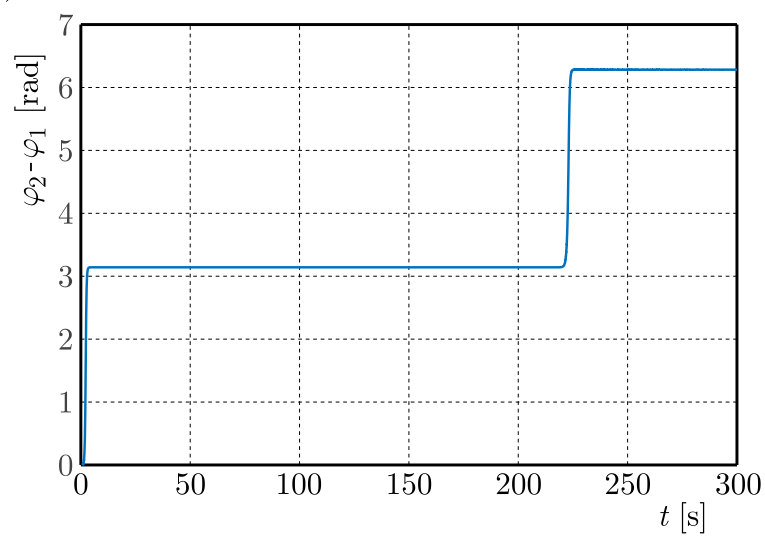

(b)

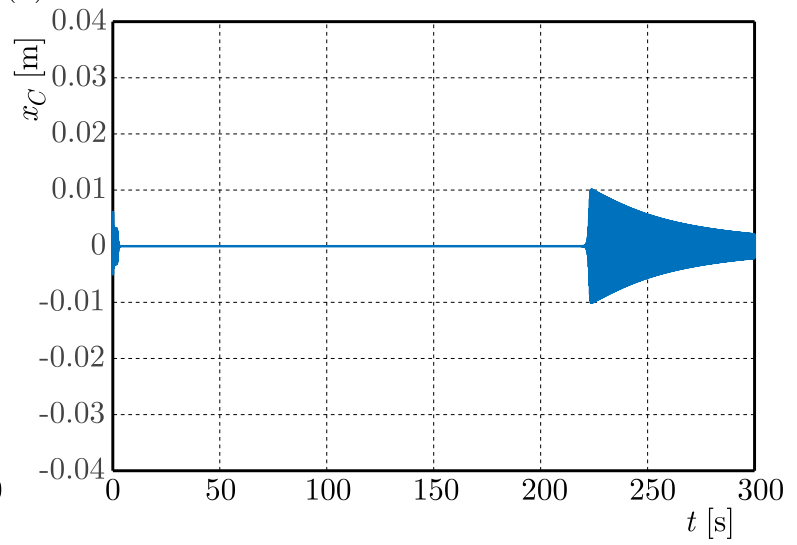

(d)

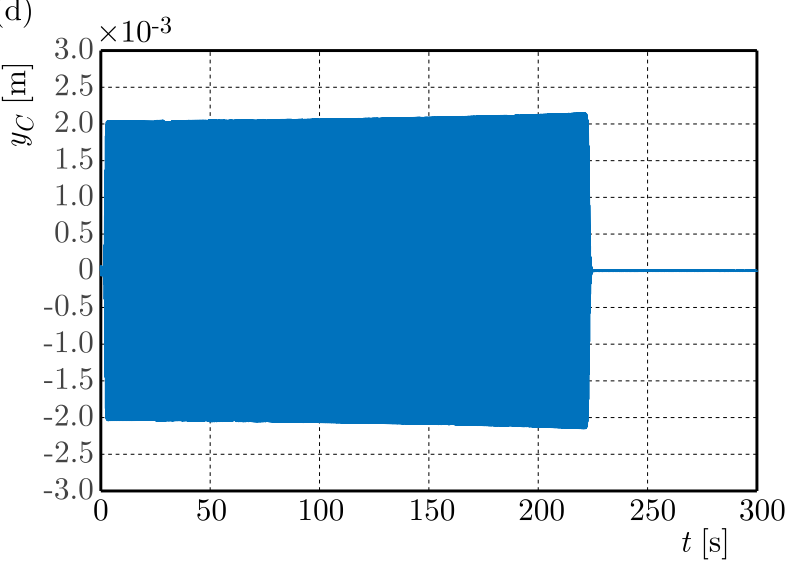

Fig. 8. Coordinates of movement of the conveyor in the case of low damping suspension, $\xi \approx 0.06$. (a) Angular velocity of the vibrator described by the coordinate $\varphi_{1}$; (b) course of the difference in coordinates of angular vibrators; course of (c) $x_{C}$ coordinate and (d) $y_{C}$ coordinate of the center of mass of the conveyor body

\section{Synchronization moment}

An equally important issue as the conditions of synchronization of the vibrators is the issue of the synchronizing moment. Its value determines the resistance of the vibrators to falling out from the position of equilibrium and the angle of phasing-out associated with the quality of transport.

The synchronizing moment can be determined on the basis of the components of vibrators equations of motion responsible for the coupling with the body. In the form after rejection of small orders of magnitude, these actions can be presented as vibration moments

$$
\begin{aligned}
& M_{v i b 1}=-m_{w} e \sin \left(\varphi_{1}\right) \ddot{x}_{C}+m_{w} e \cos \left(\varphi_{1}\right) \ddot{y}_{C}+m_{w} e\left[\cos \left(\varphi_{1}\right) \zeta_{0}+\sin \left(\varphi_{1}\right) \xi_{0}\right] \ddot{\Psi} \\
& M_{v i b 2}=-m_{w} e \sin \left(\varphi_{2}\right) \ddot{x}_{C}-m_{w} e \cos \left(\varphi_{2}\right) \ddot{y}_{C}+m_{w} e\left[\cos \left(\varphi_{2}\right) \zeta_{0}+\sin \left(\varphi_{2}\right) \xi_{0}\right] \ddot{\Psi}
\end{aligned}
$$

that affect individual vibrators. In practice, we are satisfied with the average for the period with the value of the synchronizing moment, i.e.

$$
M_{\text {sync }}=\frac{1}{T} \int_{0}^{T}\left(M_{v i b 2}-M_{v i b 1}\right) d t
$$

After insertion into (4.2), the acceleration formula $\ddot{x}_{C}, \ddot{y}_{C}, \ddot{\Psi}$ determined on the basis of e.g. solutions (2.15), we get its full form. 
In this paper, we have only provided a simplified relation without the influence of the suspension on the body movement. From a practical point of view, it is a case of a over resonance conveyor operation, where the working point of the vibrators is significantly (at least 4 times) distant from the natural frequency of the body-suspension system. With this assumption, we can calculate

$$
M_{\text {sync }} \approx m_{w}^{2} e^{2} L^{2} \omega^{2} \frac{m_{k}^{* 2}+4 m_{w}\left(m_{w}-m_{k}^{*}\right) \cos \beta^{2}}{m_{k}\left(J_{\eta}^{*} m_{k}^{*}-4 L^{2} m_{w}^{2} \cos \beta^{2}\right)} \sin \delta
$$

when $\delta=\alpha-\pi$. The maximum value of the synchronizing moment $M_{\text {sync }_{M A X}}$ is received for $\sin \delta=1$.

The $M_{\text {sync }_{M A X}}$ is important to ensure the technical stability of the system. According to the paper (Michalczy, 1995)

$$
M_{\text {sync }_{M A X}} \geqslant c M_{n}
$$

when $M_{n}$ means the rated moment of an asynchronous motor driving a single vibrator. Recommended values for the $c$ factor to guarantee the phase differences of no more than $\Delta \varphi$ are shown in Table 2.

Table 2. Recommended values for the factor $c$

\begin{tabular}{|l|c|c|}
\hline Machine & $c$ & $\Delta \varphi\left[^{\circ}\right]$ \\
\hline \hline Conveyor & $0.5-1.0$ & $12-16$ \\
\hline Feeder & $1.0-2.4$ & $5-12$ \\
\hline Sifter & $1.5-4.0$ & $3-5$ \\
\hline
\end{tabular}

Formula (4.3) has been tested. Five computer simulations were performed, see Fig. 9. The first simulation was based on parameters corresponding to a soft suspension that meets the

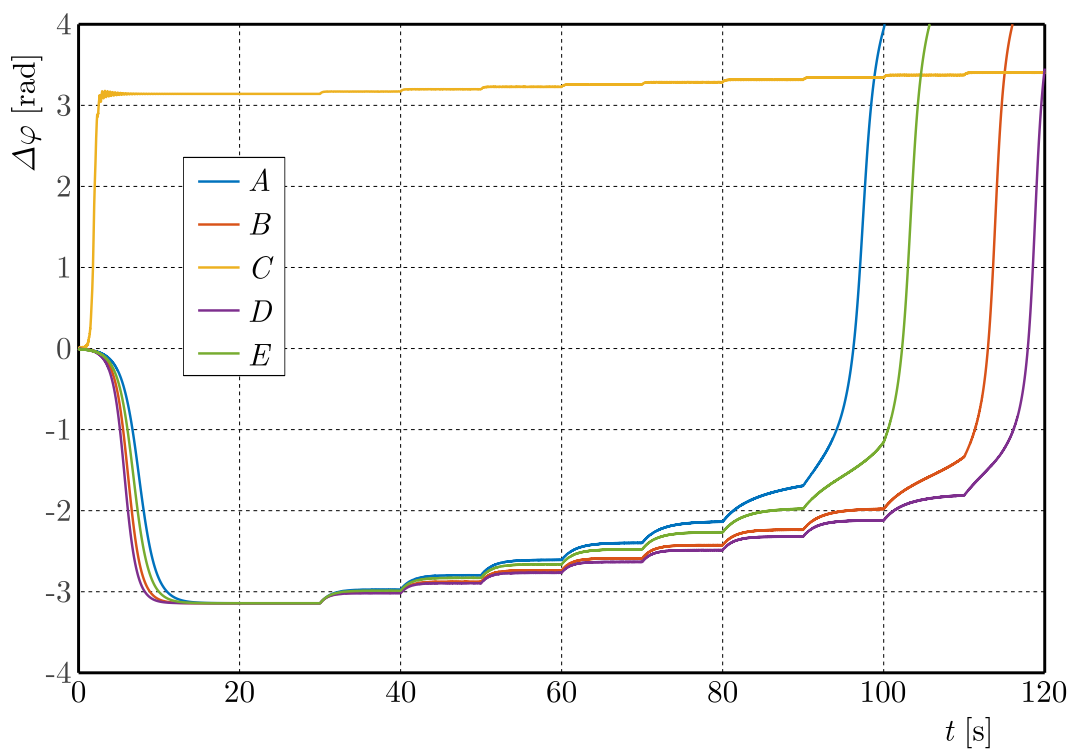

Fig. 9. Charts of the phase difference of the vibrators depending on the load; $A$ - soft suspension, $B$ - ROSTA suspension, $C$ - ROSTA AB D suspension, $D$ - ROSTA suspension and displacement of the synchronization plane below the body center of gravity, $E$ - soft suspension and displacement of the synchronization plane below the body center of gravity

requirement of at least 4 times distance between the natural frequency of the suspension-body system and the velocity of vibrators. The second was carried out on the basis of parameters of 
soft metal-elastomer suspension characterizing slim elements. The third simulation was based on the parameters of a highly directional suspension in which the elastic coefficient in the transverse direction to the working plane was increased 5 times in relation to the slim structures. The fourth and fifth correspond to simulations of the second and first, but with a lower synchronization plane of the vibrators in relation to the centre of gravity of the machine body.

The factor that pulls the vibrators out of the synchronism is the systematically increased load moment $\Delta M_{\text {load }}$ applied to the rotor shafts as follows

$$
M_{\text {load }_{1}}=+\frac{\Delta M_{\text {load }}}{2} \quad M_{\text {load }_{2}}=-\frac{\Delta M_{\text {load }}}{2}
$$

In simulations presented in the graph, the loading moment was activated in 30 seconds of the simulation time and increased by $0.1 \mathrm{Nm}$ every 10 seconds. According to formula (4.3) and the parameters given in Table 7 , the value of $M_{\text {sync }}$ MAX $=0.54 \mathrm{Nm}$.

It can be observed that full compatibility of the formula with the results of the simulation occurred only in the first case; in the time interval of $80 \mathrm{~s}-90 \mathrm{~s}$ the loading moment was $0.6 \mathrm{Nm}$, and in this interval the vibrators were disassembled. On the basis of other simulations, we can see that the synchronizing bond between the vibrators was stronger, and the systems with stiffer suspensions showed a higher synchronizing moment. Also, the displacement of the synchronization plane of the vibrators below the center of gravity resulted in an increase in the synchronizing moment. This situation can be explained by an increase in the amplitude of vibrations caused by the shift of resonances in the direction of the working point or by activation of other motion coordinates.

\section{Conclusions}

The paper presents the analysis of a self-synchronization process of inertial vibrators of a vibratory conveyor in which the self-synchronization plane of vibrators is perpendicular to the working plane of the conveyor trough. It shows that in the case of a conveyor of typical construction, symmetrically in relation to the vertical plane passing along the length of the conveyor and where the plane of synchronisation of the vibrators passes through the center of gravity of the body, the system may be considered as a plane one. The authors introduce a function on the basis of which it is possible to determine frequency ranges of operation of rotors for which the desired synchronization occurs, i.e. where the vibrators produce a sinusoidal force passing through the center of mass of the machine body along the conveyor trough. The autors analyzed the influence of stiffness of the suspension on the synchronization plane. It is proved that in the case of suspensions in which there is a significant difference of elastic coefficients in the main directions, there is a danger of transition from the desired to undesired synchronization. We suggest that in such a case, we can apply metal-elastomer suspensions. The danger of the transition from the desired to undesired synchronization state can be increased by using frequency inverters that reduce velocity of the drive motors. We notice the influence of damping on reduction of the number of switches between the state of desired and undesired synchronization.

An approximate formula for the value of the synchronizing moment (omitting of the suspension components) has also been derived. The formula may by applied to conveyors working over-resonance meeting the requirement of multiple (at least 4 times) difference in the frequency of free vibrations of the suspension-body system in relation to the rotation speed of vibrators. On the basis of simulation tests performed on the full model of the conveyor, a positive effect was found of the shift of the conveyor free vibration frequency towards the angular velocity of the vibrators, as well as the movement of the synchronization plane of the vibrators below the center of gravity of the body. These facts can be explained by an increase in the amplitude of vibration of the body. In the first case, the phenomenon is caused by the shift of the resonance 
towards the working point. In the second, the appearance of motion in additional coordinates. However, the increase in the synchronization moment obtained through these paths has negative influence on the uniformity of motion of the conveyor trough and the quality of transport associated with it.

\section{Acknowledgement}

The work described in this paper has been performed under project No. 11.11.130.734.

\section{References}

1. Blekhman I.I., 1971, Synchronization of Dynamic Systems, Nauka Press, Moscow

2. Blekhman I.I., 2000, Vibrational Mechanics: Nonlinear Dynamics Effects, General Approach, Applications, World Scientific Publishing Co. Pte. Ltd.

3. Cieplok G., 2009, Verification of the nomogram for amplitude determination of resonance vibrations in the run-down phase of a vibratory machine, Journal of Theoretical and Applied Mechanics, 47, 2, 295-306

4. Cieplok G., 2018, Estimation of the resonance amplitude in machines with inertia vibrator in the coast-down phase, Mechanics and Industry, 19, 1, 1-9

5. FAng P., Hou Y., NAN Y., 2015, Synchronization of two homodromy rotors installed on a double vibro-body in a coupling vibration system, PLOS ONE, 10, 5, 1-22,

6. Francke M., Pogromsky A., Nijmeijer H., 2020, Huygens' clocks: sympathy and resonance, International Journal of Control, 93, 2, 274-281

7. Goncharevich I.F., Frolov K.V., 1990, Theory of Vibratory Technology, Hemisphere Publishing Corporation. New York, Philadelphia

8. Hou Y., Du M., Fang P., Zhang L., 2017, Synchronization and stability of an elastically coupled tri-rotor vibration system, Journal of Theoretical and Applied Mechanic, 55, 1, 227-240

9. Karmazyn A., Balcerzak M., Perlikowski P., Stefanski A., 2018, Chaotic synchronization in a pair of pendulums attached to driven structure, International Journal of Non-Linear Mechanics, 105, 261-267

10. MichalczyK J., 1995, Vibrating Machines. Dynamic Calculations, Vibrations, Noise (in Polish), Wydawnictwo Naukowo-Techniczne, Warszawa

11. MichalczyK J., 2012, Inaccuracy in self-synchronisation of vibrators of two-drive vibratory machines caused by insufficient stiffness of vibrators mounting, Archives of Metallurgy and Materials, $\mathbf{5 7}, 3,823-828$

12. MichalcZYK K., 2017, Natural transverse vibrations of helical springs in sections covered with elastic coatings, Bulletin of the Polish Academy of Sciences: Technical Sciences, 65, 6, 949-959

13. Michalczyk K., Bera P., 2019, A simple formula for predicting the first natural frequency of transverse vibrations of axially loaded helical springs, Journal of Theoretical and Applied Mechanic, 57, 3, 779-790

14. Michalczyk J., Cieplok G., 2014, Disturbances in self-synchronisation of vibrators in vibratory machines, Archives of Mining Sciences, 59, 1, 225-237

15. Michalczyk J., PakuŁa S., 2016, Phase control of the transient resonance of the automatic ball balancer, Mechanical Systems and Signal Processing, 72-73, 254-265

16. Sikora W., Michalczyk K., Machniewicz T., 2016, A study of the preload force in metal-elastomer torsion springs, Acta Mechanica et Automatica, 10, 4, 300-305

17. Sikora W., Michalczyk K., Machniewicz T., 2018, Numerical modelling of metal-elastomer spring nonlinear response for low-rate deformations, Acta Mechanica et Automatica, 12, 1, 31-37

18. Zhaо C., Zhao Q., Gong Z., Wen B., 2011, Synchronization of two self-synchronous vibrating machines on an isolation frame, Shock and Vibration, 55, 1-2, 73-90 